\title{
Large area, label-free imaging of extracellular matrix using telecentricity
}

\author{
Michelle A. Visbal Onufrak ${ }^{\mathrm{a}}$, Raymond L. Konger ${ }^{\mathrm{b}}$, Young L. Kim ${ }^{\mathrm{a}, \mathrm{c}}$ \\ ${ }^{a}$ Weldon School of Biomedical Engineering, Purdue University, West Lafayette, Indiana 47904, \\ USA; ${ }^{b}$ Department of Pathology \& Laboratory Medicine and Department of Dermatology, Indiana \\ University School of Medicine, Indianapolis, Indiana 46202, USA; ${ }^{\circ}$ Department of Computer \\ Science and Engineering, Kyung Hee University, Yongin, 446-701, South Korea
}

\begin{abstract}
Subtle alterations in stromal tissue structures and organizations within the extracellular matrix (ECM) have been observed in several types of tissue abnormalities, including early skin cancer and wounds. Current microscopic imaging methods often lack the ability to accurately determine the extent of malignancy over a large area, due to their limited field of view. In this research we focus on the development of simple mesoscopic (i.e. between microscopic and macroscopic) biomedical imaging device for non-invasive assessment of ECM alterations over a large, heterogeneous area. In our technology development, a telecentric lens, commonly used in machine vision systems but rarely used in biomedical imaging, serves as a key platform to visualize alterations in tissue microenvironments in a label-free manner over a clinically relevant area. In general, telecentric imaging represents a simple, alternative method for reducing unwanted scattering or diffuse light caused by the highly anisotropic scattering properties of biological tissue. In particular, under telecentric imaging the light intensity backscattered from biological tissue is mainly sensitive to the scattering anisotropy factor, possibly associated with the ECM. We demonstrate the inherent advantages of combining telecentric lens systems with hyperspectral imaging for providing optical information of tissue scattering in biological tissue of murine models, as well as light absorption of hemoglobin in blood vessel tissue phantoms. Thus, we envision that telecentric imaging could potentially serve for simple site-specific, tissue-based assessment of stromal alterations over a clinically relevant field of view in a label-free manner, for studying diseases associated with disruption of homeostasis in ECM.
\end{abstract}

Keywords: light propagation in tissues, tissue optical properties, diffuse light suppression, tissue anisotropy, telecentricity, hyperspectral, label-free, large area imaging

\section{INTRODUCTION}

Microstructural alterations in the extracellular matrix (ECM) (e.g. collagen matrix remodeling and realignment) have been observed in some types of tissue abnormalities including early cancer and wounds. In particular, neoplastic transformation (i.e. conversion of a normal tissue into a malignant tumor) is highly linked to changes in ECM in the stromal tissue ${ }^{1}$. Disruption of homeostasis in ECM can generally lead to fibrotic diseases, such as pulmonary fibrosis, systemic sclerosis, liver cirrhosis, cardiovascular diseases, and even cancer ${ }^{2}$. For instance, the formation of a carcinogenic field (i.e. tumor 'hotspot') at a premalignant stage takes place via alterations in tissue microenvironments, often in a heterogeneously large area. Current optical microscopy methods (e.g. multiphoton or second harmonic generation microscopy) have shown morphological and organizational alterations in $\mathrm{ECM}^{3-5}$. However, these cannot be easily adapted onto clinical settings, mainly due to their limited field of view. Indeed, although these advanced optical imaging technologies are promising, a critical limitation for widespread clinical adaptation is that they only allow sampling of a small fraction of tissue under investigation. Thus, examining a small area using conventional microscopic techniques is not appropriate to obtain an adequate field assessment of clinically significant areas for diagnosis and treatment of ECM-related diseases in clinical settings. Consequently, no simple and cost-effective imaging methods are available for clinicians that can serve as accurate visual guidance for devising an effective treatment plan over a large affected area.

In this respect, our objective is to develop a new class of mesoscopic (i.e. between microscopic and macroscopic) biomedical imaging system, designed to non-invasively assess alterations in tissue microenvironments. In our technology development, a telecentric lens system serves as a key platform to non-invasively visualize alterations in the

Optical Biopsy XV: Toward Real-Time Spectroscopic Imaging and Diagnosis, edited by Robert R. Alfano,

Stavros G. Demos, Proc. of SPIE Vol. 10060,1006010 - ( ) 2017 SPIE · CCC code: $1605-7422 / 17 / \$ 18$ doi: $10.1117 / 12.2253388$ 
tissue microenvironments over a clinically relevant tissue area. A telecentric lens is a compound lens system modified with an additional aperture for achieving constant magnification and focus over a long distance. Telecentric lenses can remove the parallax error that makes closer objects appear to be larger than objects farther from the lens. ${ }^{6}$ The use of telecentric lenses has been primarily noted in the manufacturing industry where they are integrated into machine vision systems for defect recognition during assembly lines and package inspections. We note that there were a few successful attempts to use telecentric lenses for optical computed and emission tomography. ${ }^{7-9}$ However, the advantage of telecentric lenses for imaging highly anisotropic scattering medium, specifically biological tissue had not yet been explored systemically. Recently, we have proven the advantages of using telecentric lens for imaging through highly scattering media such as biological tissue. ${ }^{10,11}$ In this respect, we introduce that a telecentric lens can provide a simple mesoscopic (between microscopic and macroscopic) imaging platform for removing diffuse light in tissue imaging.

\section{DIFFUSE LIGHT SUPPRESSION USING TELECENTRIC LENS}

\subsection{Background}

In a recent study, we took advantage of a telecentric lens system to easily implement directional angular gating in a reflection mode of optical tissue imaging, which can discard a relatively significant amount of unwanted scattered or diffuse light in highly anisotropic biological tissue. First, we characterized the telecentricity (i.e. acceptance angle for image formation) of a telecentric lens that allows for large-area tissue image with demagnification (e.g. $0.3 \times-0.2 \times$ ). For this, we determined the degree of telecentricity of the telecentric lens (Schott Moritex Co.) that is capable of mesoscopic tissue imaging in a field of view of $50 \mathrm{~mm}$ in diameter, using a telecentricity target (Edmund Optics, Inc.) We hypothesized that with a small acceptance angle of $0.55^{\circ}$, the telecentric lens can remove off-axis unwanted diffuse light in a reflectance mode. Then, because the exit angle of the light diffused from biological tissue at its surface is large, the telecentric lens can act as back-directional angular gating for simply discarding a portion of off-axis unwanted diffuse light in the highly anisotropic scattering medium.

To implement the telecentric lens for reflectance imaging of tissue and compare with conventional non-telecentric imaging, we built mesoscopic imaging systems employing either a telecentric or a conventional lens. In order to make an accurate comparison of the illumination to a sample using conventional lens, we used diffuse light via a ring illuminator. When the illumination output beam was at a proper distance to achieve a focused image for either telecentric or conventional lens, the divergence angle of the illumination beam was $\sim 0.9^{\circ}$ for the ring illuminator. For both systems, the field of view was $\sim 60 \mathrm{~mm} \times 60 \mathrm{~mm}$ with a pixel size of $\sim 77 \mu \mathrm{m}$. A minor non-uniformity of the illumination on the sample was also compensated by normalizing with a reference measurement from a reflectance standard.

\subsection{Imaging through biological tissues}

As representative biological tissue, we utilized SKH-1 hairless mouse $\operatorname{skin}^{12}$ as scattering media with a black and white edge image placed underneath, and attempted to resolve the black and white edge that was now embedded under the mouse skin, as shown in Figure 1. In this case where a target object is embedded in a scattering medium, the enhancement of the image resolution of the target provides direct information on how much unwanted diffuse light is removed. The scattering properties of the mouse skin tissue were determined using an integrating sphere method: ${ }^{12,13}$ (i) scattering mean free pathlength (i.e. average distance of a single scattering event) $l_{S}=81.5 \pm 12.5$ (standard deviation) $\mu \mathrm{m}$; (ii) anisotropy factor $g=0.84 \pm 0.04$; and (iii) optical thickness $\tau=12$ at $632 \mathrm{~nm}$. From grayscale images obtained from the two imaging configurations, we characterized the resolving power of the edge target by computing image resolution using the knife edge method. ${ }^{14}$ The horizontal intensity crossing over the edge from the dark to the light was averaged vertically, to represent the edge spread function (ESF), as shown in Figure 1. To compensate for speckle-like noise, ESF was fitted with a Fermi function such that:

$$
\operatorname{ESF}(x)=\sum_{i=0}^{2} \frac{a_{i}}{e^{\left[\left(x-b_{i}\right) / c_{i}\right]}+1}+d,
$$

where $a_{i}, b_{i}, c_{i}$, and $d$ are constants. We obtained a line spread function (LSF) by taking the derivative of ESF with respect to $x$ :

$$
\operatorname{LSF}(x)=\left|\frac{d E S F(x)}{d x}\right|
$$


Then, the resolving power of the edge target was calculated with a full width at half maximum (FWHM) of LSF as the image resolution, showing that the telecentric system has an image resolution of $13.9 \mathrm{~mm}$, which is approximately $25 \%$ lower than that of the conventional non-telecentric system with a FWHM of $17.6 \mathrm{~mm}$.

Telecentric Lens

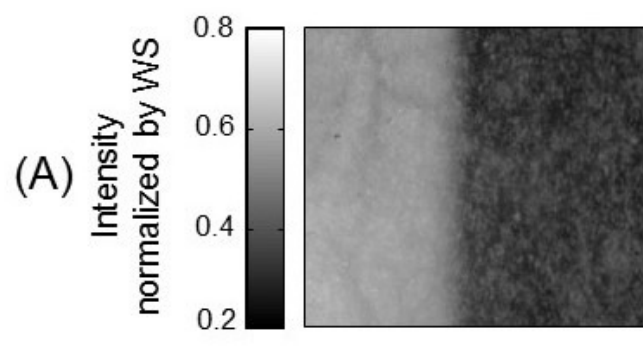

(B)

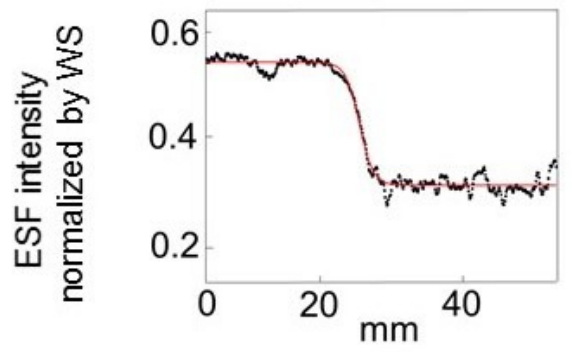

(C)

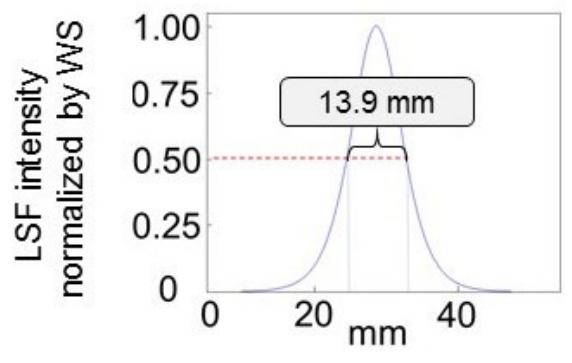

\section{Conventional Lens}
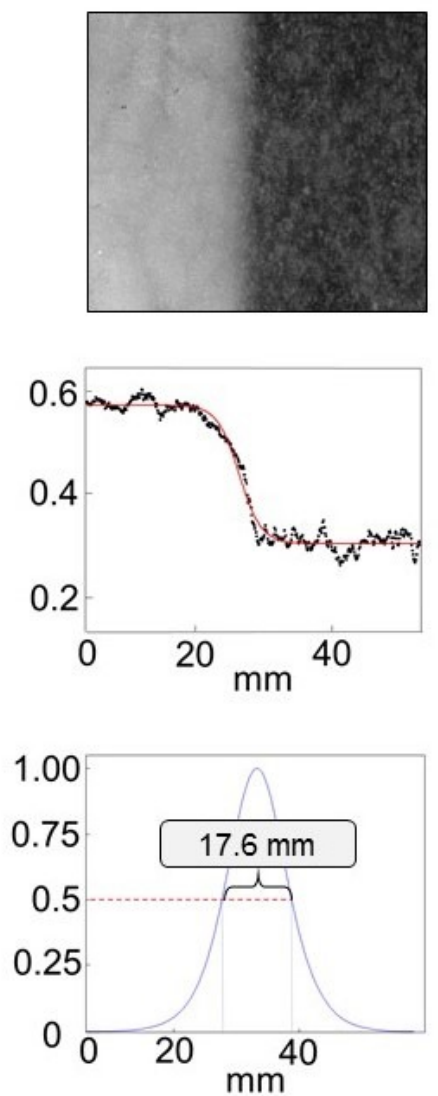

Figure 1. Planar imaging of white and black edge targets embedded in biological tissue (hairless mouse skin) using telecentric or conventional imaging system configurations. (A) Grayscale images of the edge target underneath the mouse skin. Images are normalized by a reference image obtained from a white reflectance standard (WS). (B) Averaged edge spread function (ESF) of the edge target. ESF was fitted with a Fermi function to reduce noise. (C) Calculated line spread function (LSF). FWHM is calculated to quantify the resolving power of the embedded target as an image resolution.

\subsection{Tissue phantoms}

We also conducted a series of tissue phantoms to investigate the effect of $g$ on the image resolution of an embedded target, using telecentric imaging versus conventional imaging. In particular, we compared resolution enhancement, resulting from diffuse light suppression, as a function of $g$ while keeping other scattering properties (i.e. $l_{S}$ and $\tau$ ) fixed. For this, we prepared four tissue phantoms with different $g$ using aqueous suspensions of polystyrene microspheres (Polysciences Inc.) for $g=0.51,0.76,0.85$ and 0.90 at $\lambda=550 \mathrm{~nm}$, while $l_{S}=150 \mu \mathrm{m}$ and $\tau=15$ were kept identical for each $g$. The scattering properties of the tissue phantoms were calculated using Mie theory ${ }^{15,16}$. The scattering media of $g$ $=0.85-0.90$ are considered to be close to most biological tissue in particular to the skin. ${ }^{17,18}$ 


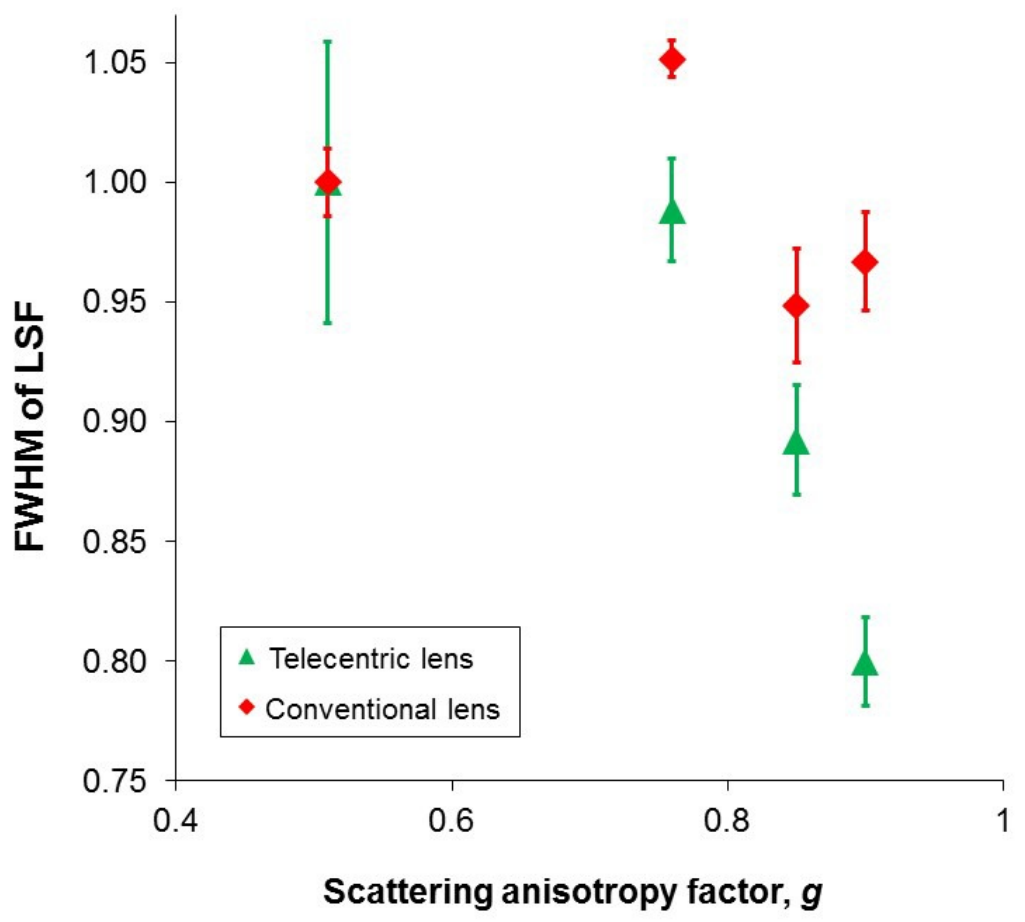

Figure 2. Effect of $g$ on the resolving power of an embedded target, using telecentric imaging versus conventional imaging. FWHM of LSF (i.e. image resolution of the target) is normalized with the largest FWHM obtained with the lowest value of $g=0.51$ to compare the sensitivity to $g$ under different system configurations. The relative enhancement of image resolution of the embedded target provides information on the level of unwanted diffuse light removal.

To carry out similar imaging tests, we replaced the mouse skin with different tissue phantoms prepared to mimic biological scattering media and placed them over a white and black edge image. For each $g$, at least six different tissue phantoms were prepared and imaging tests were repeated independently. Overall, as $g$ increases, more clear distinctions between the white and black edge images are seen from telecentric imaging due to the relative suppression of unwanted diffused light. On the other hand, the edge images are relatively invariable without strongly depending on $g$ for conventional non-telecentric imaging. Similar to the analyses in the previous example using the mouse skin, we computed image resolutions for sample replicates with different $g$ under the two system configurations. In Figure 2, the telecentric imaging system shows rapid decreases in FWHM of LSF (i.e. resolution enhancement) as $g$ approaches to the typical values of biological tissue. However, the conventional non-telecentric system is not dependent on $g$ with no statistical significance (i.e. p-value for the slope between FWHM of LSF and $g=0.19$ ). In other words, only backscattered images acquired by the telecentric lens were sensitive to changes in $g$, in comparison to its conventional lens imaging counterpart. These results support the idea of simple yet effective removal of a portion of diffuse light in biological tissue using telecentric imaging.

\subsection{Discussion}

Telecentric lens presents an advantage of resolution enhancement over conventional lens for imaging of an embedded object through scattering medium, such as biological tissue. As portrayed in the results from our study using skin excised from SKH mouse, a significantly lower resolution was obtained for the telecentric configuration when compared to imaging using a conventional lens. This was in agreement with resolution phantom studies of higher anisotropy factors in the biological tissue range of $0.85-0.9$. From the resolution results of our tissue-mimicking phantom studies we observed that telecentric lens imaging is sensitive to changes in anisotropy factor of the imaging medium, when compared to conventional lens imaging where no statistically significant differences were obtained. 


\section{SPECTROMETER-LESS TELECENTRIC IMAGING SYSTEM}

\subsection{Motivation}

While demonstrating that a telecentric lens system can act exactly as back-directional (angle) gating (or filtering), allowing to take advantage of the intrinsic properties of biological tissue to enhance the field of view and image resolution $^{10}$, we proposed to develop a telecentric imaging platform, which would also provide for a large imaging area, as well as a lightweight and compact system for easy operation. Now, in order to achieve label-free imaging, hyperspectral imaging is widely used in providing information of light absorbers in biological tissue. Conventional hyperspectral imaging systems rely on the use of a mechanical filter wheel, an imaging spectrograph, or a liquid crystal tunable filter. One alternative to overcome this restriction would be to implement a spectrometer-less imaging system. This can be achieved by utilizing an algorithm that can reliably reconstruct full spectral information from RGB image data, which are easily acquired by commonly used three-color CCD (or CMOS) cameras. Using this simpler technique, spectral information could be extracted from RGB data in color images. ${ }^{11}$ With this in mind, we propose to reconstruct multispectral information from red green blue (RGB) images acquired by a color CMOS camera ${ }^{19}$. The 3-color CMOS allows us to acquire a simplified data set of (x,y, and wavelength $\lambda$ ) for spectral analyses to analyze tissue absorption from changes in hemoglobin content as well as extract tissue scattering signals owing to changes in the tissue microenvironment or extracellular matrix. Fortunately, several methods for spectral reconstruction with RGB data have been studied extensively ${ }^{20}$. These hyperspectral reconstruction methods using RGB data could potentially lead to a simplified instrumentation and operation without using bulky and expensive spectrometers.

An important consideration however, is that the reflectance data acquired is most sensitive to the illumination-collection of the system used for scanning ${ }^{21}$, therefore, it is imperative that both hyperspectral and RGB images for the purpose of data reconstruction algorithm development be acquired for the same optical position and illumination distribution for each setting. In this sense, we require a dual imaging system that can acquire each data set incurring in the least illumination angle or focus modifications possible required to alternate between imaging modes. In our work, the combination of spectroscopic measurements with diffuse light-suppressed imaging in the reflection mode was integrated into a dual-mode imaging device for visualization of blood vessels and accurate quantification of hemoglobin absorbance. This modality could be later employed for probing vascularity growth over a period of time, while embedded in a relatively thick scattering medium such as the skin, since absorption spectral analysis over a broad range of wavelengths can permit accumulation of the contrast improvement at each wavelength.

\subsection{Instrumentation Development}

The first stage of development for this dual imaging system consisted in constructing a working prototype system that would enable acquisition of both hyperspectral and RGB image information. Specifically, to produce a spectroscopic imaging scheme that acquires spectral as well as spatial information simultaneously, we constructed a working prototype of a dual imaging system. This system would serve as a platform to provide training data sets for developing a hyperspectral data reconstruction algorithm utilizing RGB image data under equal illumination conditions and imaging parameters. During our instrumentation development, we pursued two distinct approaches: (A) using a liquid tunable filter, or (B) a line-scan spectrograph.

Figure 3 illustrates corresponding diagrams for these systems. The first approach involves integration of CCD camera with a liquid crystal tunable filter (Figure 3(A)). This filter, coupled to a white LED light source via an optical fiber, is used to change the wavelength from 400 to $720 \mathrm{~nm}$. The wavelength filter position is controlled via a Virtual Instrument developed using LabVIEW (National Instruments, Inc.), and the image acquisition is timed according to the filter wavelength sweep. With this Virtual Instrument, we can easily modify camera parameters in order to take either grayscale images for Hyperspectral Imaging mode, or RGB images for RGB imaging mode. In this imaging setup, image acquisition for hyperspectral or RGB must be done separately, in other words, one imaging mode must be done first since the 3-color camera sensor needs to be readjusted between greyscale or RGB mode using the Virtual Instrument. Therefore, the main drawback of this approach is that the total image acquisition time takes approximately 5 minutes total, which could affect the image quality since it is susceptible to human subject involuntary movement through this period. In addition, a liquid tunable filter represents an expensive component that is often difficult to integrate into an accessible imaging system such as mobile imaging devices. For this reason, it is preferred to have an alternative to relying on an external filter for providing illumination for all different wavelengths in order to acquire spectroscopic measurements. 
A - Using a liquid tunable filter

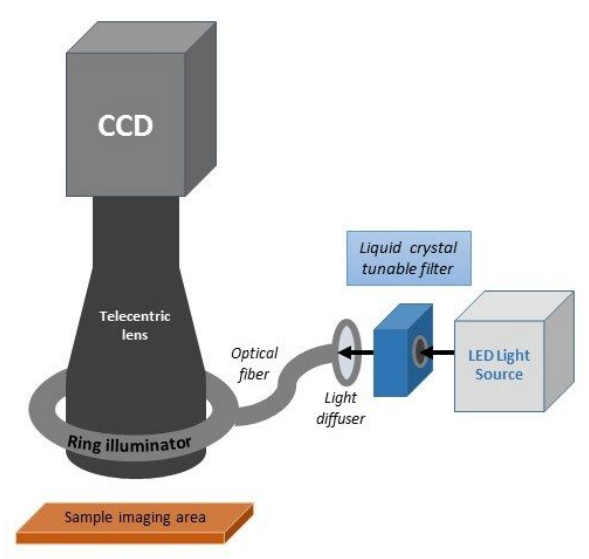

(1)

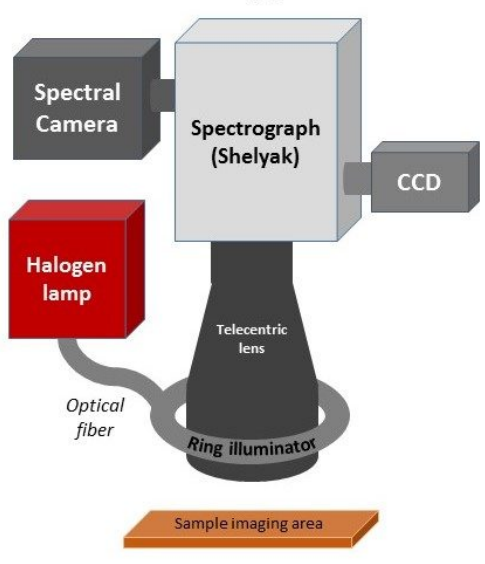

(2)

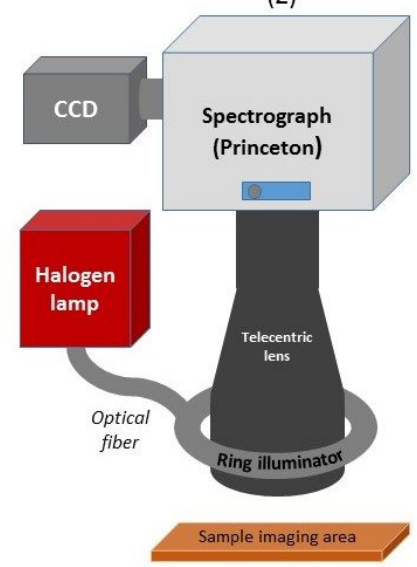

Figure 3. (A) Telecentric imaging configurations (A) utilizing a liquid crystal tunable filter (LCTF) to provide hyperspectral information. Illumination is provided by a white LED light source coupled to an optical fiber, which passes through the LCTF and light diffuser, to a ring illuminator to provide diffuse illumination to the sample imaging area under the telecentric lens. (B)(1) utilizing a dual-channel spectrograph for simultaneous acquisition of Hyperspectral and RGB data from the same (2) utilizing a Princeton Instrument model spectrograph. This spectrograph only possesses a single imaging port but its slit width and position can be easily adjusted. The spectrograph grating position can be adjusted remotely via software control. Illumination is provided by a broadband Halogen lamp, to cover both visible and near-infrared range.

Figure 3.B shows two iterations of line-scan systems. Figure 3.B(1) is assembled for simultaneous acquisition of hyperspectral and RGB data. The system consists of a dual-channel imaging spectrograph (Shelyak Instruments) with three main ports: detection port mounted with the telecentric lens (Edmund Optics), hyperspectral line-scan port mounted with the CCD camera (Princeton Instruments), and RGB image port mounted with the 3-color sensor (RGB camera, Point Grey). The spectrograph slit is a small, horizontal aperture and whose width can be adjusted to control the amount of light collected from the sample. We selected a slit width of $23 \mu \mathrm{m}$ for an acceptable spectral resolution. As for the overall imaging area, this was determined by the height of the slit and the camera sensor size. A broadband halogen lamp (Thorlabs) provides uniform illumination onto the sample via a ring illuminator around the telecentric lens. For hyperspectral line-scanning, the telecentric lens collects light scattered from the sample, which passes through the slit of the spectrograph and disperses via a diffraction grating. By adjusting the diffraction grating, the spectral range can be set for visible $(400-700 \mathrm{~nm})$ or NIR $(600-1100 \mathrm{~nm})$, with a spectral resolution of $3 \mathrm{~nm}$ and hyperspectral line-scanning distance of $6 \mathrm{~mm}$, with a pixel size of $35 \mu \mathrm{m}$. For RGB imaging, the light from the sample is also reflected via another mirror towards the RGB image port for generating an image in the visible region. The dual data set (i.e. hyperspectral line-scan and RBG image data) from the sample is normalized with a reference reflectance standard (Labsphere) to compensate for each spectral response of the dual imaging system. One advantage of RGB imaging is that RGB images are first used as guidance for locating the corresponding image site. Then, via the same telecentric lens, hyperspectral line-scanning is performed for the identical imaging area, under exact illumination conditions as for the RGB image.

We also tested a second spectrograph with a much larger slit height (Acton SP 2150, Princeton Instruments). Figure 3. $\mathrm{B}(2)$ shows a schematic of the telecentric imaging system utilizing this spectrograph. The advantage to this spectrograph model is that its slit height is sufficiently large to cover the entire height of the imaging area of the camera sensor size. Although this second spectrograph does not possess dual imaging ports, it does have an interchangeable dual grating turret in the spectrograph which allows us to mount a mirror and a diffraction grating (groove density $=300$ $\mathrm{g} / \mathrm{mm}$ ) for two different acquisition modes. In this manner, it can be easily adjusted via control software, in order to cover different wavelengths ranging from visible to near-infrared (i.e. $400 \mathrm{~nm}-1038 \mathrm{~nm}$ ), as well as a mirror position in order to acquire the RGB image. The slit in the spectrograph can also be moved from the lens light path, so when the grating is exchanged to mirror position inside the spectrograph, the system allows image acquisition for the entire imaging area for the sample under the lens, acting not only as a guidance for line-scan but also as the RGB image of interest. This system has an imaging area of $25^{2} \mathrm{~mm}^{2}$ with a pixel size of $24 \mu \mathrm{m}$. A white-light image contains all of the wavelengths from $400 \mathrm{~nm}$ to $1038 \mathrm{~nm}$, which are determined by the spectral response of the system including the light source and the CCD camera. Table 1 summarizes general imaging performance parameters for all three different 
configurations reported, where the last two correspond to different types of line-scan spectrographs. For instance, configuration A (liquid tunable filter) can be useful to image over a large area comprising several centimeters, however given the larger image collection time required, it is more sensitive to image noise coming from human subject involuntary movement, as well as a limited wavelength resolution. In contrast, configuration using a line-scan spectrograph has improved wavelength resolution, yet this approach would be most useful for imaging over smaller tissue areas, such as for detection of blood microvessels.

Table 1. Comparison of parameters for the different dual hyperspectral and RGB imaging systems.

\begin{tabular}{|c|c|c|c|}
\hline \multirow{2}{*}{$\begin{array}{c}\text { Spectral scanning } \\
\text { method }\end{array}$} & $\begin{array}{c}\text { (A) } \\
\text { Liquid } \\
\text { Specifications: }\end{array}$ & \multicolumn{2}{|c|}{ (B) } \\
\cline { 3 - 4 } & Lunable filter & $\begin{array}{c}\text { 1-Simultaneous } \\
\text { hyperspectral \& } R G B\end{array}$ & $\begin{array}{c}\text { 2-Adjustable } \\
\text { grating position }\end{array}$ \\
\hline Spectral resolution & $10 \mathrm{~nm}$ & $3 \mathrm{~nm}$ & $3 \mathrm{~nm}$ \\
\hline Pixel size & $17 \mu \mathrm{m}$ & $35 \mu \mathrm{m}$ & $24 \mu \mathrm{m}$ \\
\hline Imaging Area / height & $\mathrm{A}=3.5 \mathrm{x} 4 \mathrm{~cm}$ & $\mathrm{~h}=6 \mathrm{~mm}$ & $\mathrm{~h}=2.5 \mathrm{~cm}$ \\
\hline Acquisition time & 5 minutes & 2 minutes & 2 minutes \\
\hline
\end{tabular}

\subsection{Imaging blood vessels through highly anisotropic media}

To evaluate the imaging ability of line-scanning and imaging of blood microvessels through a highly anisotropic media similar to biological tissues, we prepared optical tissue phantoms with blood vessels, by filling low-density polyethylene (LDPE) tubing (I.D. $=0.58 \mathrm{~mm}$ and O.D. $=0.99 \mathrm{~mm}$ ) with hemoglobin in PBS for concentrations 2.5 and $4.0 \mathrm{mg} / \mathrm{ml}$. Using the line-scan configuration (B), we acquired hyperspectral and RGB or white light images for two LDPE tubings containing different hemoglobin concentrations were fixed at the bottom of a glass petri dish. We varied the separation distance of the hemoglobin-filled tubings from 1 to $10 \mathrm{~mm}$. To include optical scattering effects, we added a suspension with $0.746-\mu \mathrm{m}$-diameter polystyrene microspheres to the petri dish until submerging the hemoglobin-filled tubing, for optical parameters close to previous tissue phantoms experiments $\left(g=0.85, l_{S}=142.7 \mu \mathrm{m}, \tau=14.3\right)$. On Figure $4(\mathrm{~A})$, the guide lines are given to indicate the position of the slit, with respect to the blood vessel phantom. To quantify Hgb content along the line, we applied a Partial Least Squares Regression (PLSR) model at each location along the line-scan to generate a hemoglobin spatial profile, which corresponds to the line marked in the RGB image. We quantified the spatial feature of the blood vessel phantoms by calculating a separation distance between the two blood vessels embedded in the phantoms. Then, we compared it with those obtained from corresponding RGB or visible images, which serve as a reference of visible imaging. Figure $4(C)$ shows a correlation of vessel distances calculated by NIR and visible imaging with $\mathrm{R}^{2}=0.998$. 


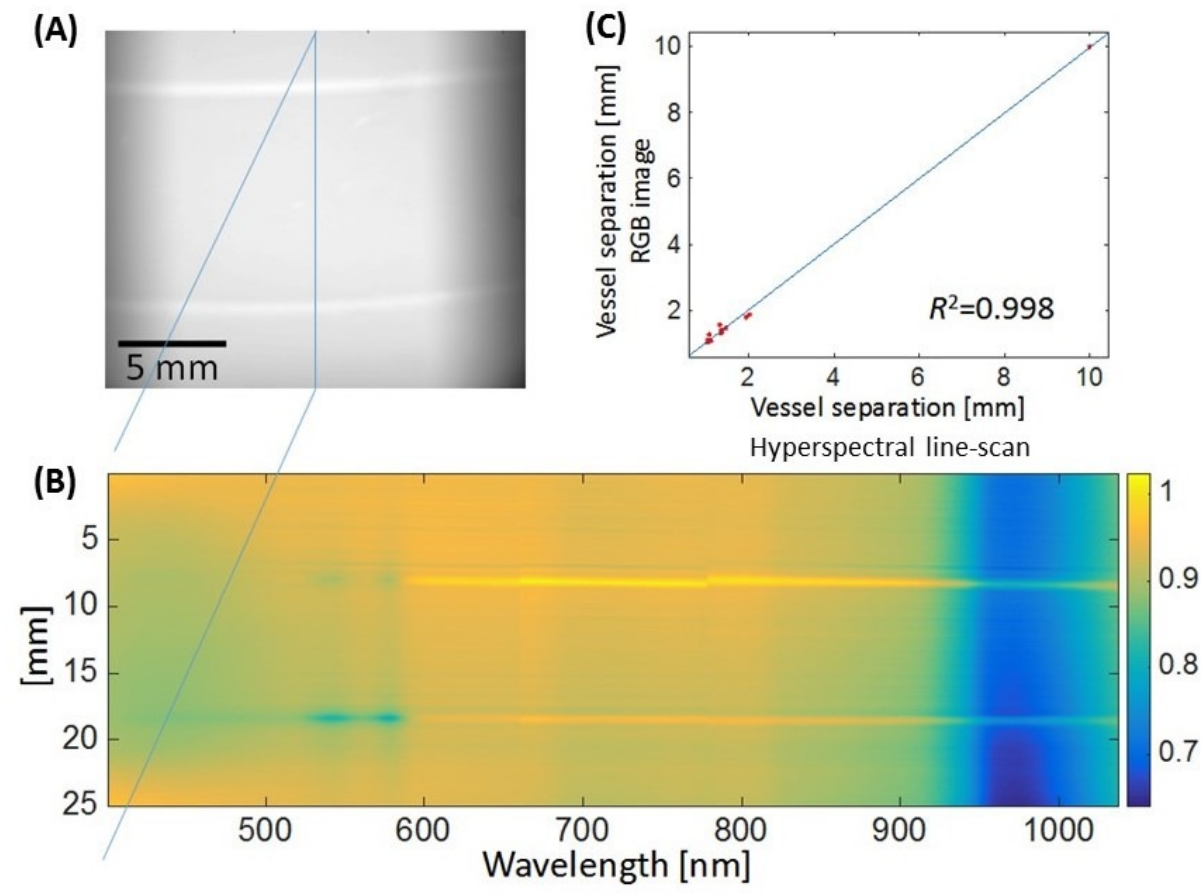

Figure 4. Comparison between visible and hyperspectral imaging. (A) Visible light image of a blood vessel phantom. The vertical line indicates the line-scanning location. (B) Spatial-spectral map of the reflectance from the same blood vessel phantom. (C) Separation distance of two blood vessels (phantoms) for comparing the two imaging schemes.

\section{CONCLUSIONS}

From the resolution results of our tissue-mimicking phantom studies we observed that telecentric lens imaging is sensitive to changes in anisotropy factor of the imaging medium, when compared to conventional lens imaging where no statistically significant differences were obtained. From these findings, we can conclude that telecentric lens can act as a back-directional angle filter, which collects the small solid angle to form an image in the exact backward direction in reflection mode. In general, i) back-directional gating effectively removes diffuse light reflected from biological tissue in a reflectance mode, because most biological tissue is highly anisotropic. ii) This also could be used as an intrinsic tissue contrast with a wide field of view, because the angular scattering distribution of tissue is highly sensitive to tissue architectures in the backward direction. A telecentric lens-based imaging system then makes it suitable for easy integration into a clinical imaging device. This would provide significant advantage over conventional optical techniques in the sense that a single telecentric lens can replace several optical components to generate 2D images. We also demonstrated that the combination of telecentric with spectrometer-less hyperspectral imaging shows promise for developing simple instrumentation and rapid image acquisition without using a bulky and expensive spectrometer. Particularly, while comparing different spectrograph configurations, it is imperative to decide the type of spectrograph with the imaging parameters required for the application of interest. Overall, our technology can serve as a platform for easy integration into an instrument of modest price and design, and of interest for potential integration with smartphones or used for health care in home-based or resource-limited settings.

\section{ACKNOWLEDGEMENTS}

This work was supported by the SAMSUNG Global Research Outreach (GRO) Program. 


\section{REFERENCES}

[1] Allen M, Louise Jones J, "Jekyll and Hyde: the role of the microenvironment on the progression of cancer," J Pathol 223(2), 162-76 (2011)

[2] Cox TR, Erler JT "Remodeling and homeostasis of the extracellular matrix: implications for fibrotic diseases and cancer," Disease models \& mechanisms 4(2), 165-78 (2011)

[3] Zoumi A, Yeh A, Tromberg BJ, "Imaging cells and extracellular matrix in vivo by using second-harmonic generation and two-photon excited fluorescence," Proceedings of the National Academy of Sciences 99(17), 110149 (2002)

[4] Schenke-Layland K, "Non-invasive multiphoton imaging of extracellular matrix structures," Journal of Biophotonics 1(6), 451-62 (2008)

[5] Campagnola P, "Second harmonic generation imaging microscopy: applications to diseases diagnostics," Analytical Chemistry 83(9), 3224-31 (2011)

[6] Watanabe M., \& Nayar S K., "Telecentric optics for focus analysis," IEEE Transactions on Pattern Analysis and Machine Intelligence 19(12), 1360-1365 (1997)

[7] Sakhalkar H S, Oldham M, "Fast, high-resolution 3D dosimetry utilizing a novel optical-CT scanner incorporating tertiary telecentric collimation," Med Phys 35(1), 101-111 (2008)

[8] Thomas A, Bowsher J, Roper J, Oliver T, Dewhirst M, Oldham M, "A comprehensive method for optical-emission computed tomography," Phys Med Biol 55(14), 3947-3957 (2010)

[9] Thomas A, Newton J, Oldham M, "A method to correct for stray light in telecentric optical-CT imaging of radiochromic dosimeters," Phys Med Biol 56(14), 4433-4451 (2011)

[10] Visbal Onufrak MA, Konger RL, Kim YL, "Telecentric suppression of diffuse light in imaging of highly anisotropic scattering media," Optics Letters 41(1), 143-146 (2016)

[11] Kim T, Kim J, Visbal Onufrak MA, Chapple C, Kim YL, "Nonspectroscopic imaging for quantitative chlorophyll sensing," Journal of Biomedical Optics 21(1), 016008 (2016)

[12] Konger R L, Xu Z, Sahu R P, Rashid B M, Mehta S R, Mohamed D R, Kim Y L, "Spatiotemporal assessments of dermal hyperemia enable accurate prediction of experimental cutaneous carcinogenesis as well as chemopreventive activity," Cancer Res 73(1), 150-159 (2013)

[13] Prahl S A, van Gemert M J C, Welch A J, "Determining the optical properties of turbid media by using the addingdoubling method," Applied Optics 32(4), 559-568 (1993)

[14] Tzannes, A P, Mooney J M, "Measurement of the modulation transfer function of infrared cameras," Optical Engineering 34(6), 1808-1817 (1995)

[15] Cheong W F, Prahl S A, Welch A J, "A Review of the Optical-Properties of Biological Tissues," IEEE Journal of Quantum Electronics 26(12), 2166-2185 (1990)

[16] Samatham R, Jacques S L, Campagnola P J, "Optical properties of mutant versus wild-type mouse skin measured by reflectance-mode confocal scanning laser microscopy (rCSLM)," Journal of Biomedical Optics 13(4), 041309 (2008)

[17] Kim Y L, Liu Y, Wali R K, Roy H K, Goldberg M J, Kromin A K, Backman V, "Simultaneous measurement of angular and spectral properties of light scattering for characterization of tissue microarchitecture and its alteration in early precancer," IEEE Journal of Selected Topics in Quantum Electronics 9(2), 243-256 (2003)

[18] van de Hulst H C, "Light scattering by small particles," New York: Dover Publications (1995)

[19] Gebhart S C, Majumder S K, Mahadevan-Jansen A, "Comparison of spectral variation from spectroscopy to spectral imaging," Applied Optics 46(8), 1343-1360 (2007)

[20] Lu G, Fei B, "Medical hyperspectral imaging: a review," Journal of Biomedical Optics 19, 010901 (2014)

[21] Shen H L, Xin J H and Shao S J, "Improved reflectance reconstruction for multispectral imaging by combining different techniques," Optics Express 15(9), 5531-5536 (2007) 